Research Paper

\title{
The value of long noncoding RNA CASC2 as a biomarker of prognosis in carcinomas: a meta-analysis
}

\author{
Xin Yan, Yanting Zhu, Fangwei Li, Wenhua Shi, Jian Wang, Qingting Wang, Qianqian Zhang, Limin Chai, \\ Manxiang Li ${ }^{\bowtie}$ \\ Department of Respiratory and Critical Care Medicine, The First Affiliated Hospital of Xi'an Jiaotong University, Xi'an, Shaanxi 710061, China \\ $\triangle$ Corresponding author: Dr. Manxiang Li, Department of Respiratory and Critical Care Medicine, the First Affiliated Hospital of Xi'an Jiaotong University, No. \\ 277, West Yanta Road, Xi'an, Shaanxi 710061, China. Telephone: +86-029-85324053; Fax: +86-029-85324053; E-mail address: manxiangli@hotmail.com \\ (c) Ivyspring International Publisher. This is an open access article distributed under the terms of the Creative Commons Attribution (CC BY-NC) license \\ (https://creativecommons.org/licenses/by-nc/4.0/). See http://ivyspring.com/terms for full terms and conditions.
}

Received: 2018.04.03; Accepted: 2018.08.26; Published: 2018.10.05

\begin{abstract}
Lnc RNA Cancer Susceptibility Candidate 2(CASC2) has been shown to be aberrantly expressed in multiple types of cancer and might serve as a prognosis biomarker. The present meta-analysis was conducted to investigate whether the expression of CASC2 was associated with prognosis or clinicopathological features in correlative cancers. A total of 11 studies with 765 cancer patients were included by searching the electronic databases, the results found a significant association between high expression of CASC2 and longer OS in cancer patients ( $\mathrm{HR}=0.43,95 \% \mathrm{Cl}: 0.33-0.55$, $P=0.000)$. In addition, a significant correlation was observed between high level of CASC2 and earlier TNM stage(OR $=0.30,95 \% \mathrm{Cl}=0.21-0.43, P<0.001)$, smaller tumor size $(O R=0.28,95 \% \mathrm{Cl}$ $=0.12-0.66, P=0.004)$, better tumor differentiation $(\mathrm{OR}=0.42,95 \% \mathrm{Cl}=0.27-0.66, P=0.0002)$. In conclusion, CASC2 can serve as a novel marker predicting the prognosis and clinicopathological features in various cancers.
\end{abstract}

Key words: CASC2, cancer, biomarker, prognosis, meta-analysis

\section{Introduction}

Cancer is a severe public health problem worldwide and the second leading cause of death around the world. It is estimated that about 1,735,3500 new cancer cases and 60,9640 cancer deaths are projected to burst in the United States in 2018[1]. Besides, it is prone to be a major cause of morbidity and mortality in the next decades worldwide[2]. Up to now, the mechanisms of oncogenesis have not been completely clarified due to its intricate process. More and more scientists have attached importance to biomarkers in cancer due to their diversified functions. Apart from early diagnosis, specific biomarkers can also play a role in precise therapy and prognostication of cancer[3].

Long noncoding RNAs are a heterogeneous class of RNAs that are longer than 200 nucleotides with restricted protein-coding capacity[4]. LncRNAs are implicated in manifold functions, including cellular trafficking, immune surveillance, epigenetic regulation, splicing and pluripotency of embryonic stem cells and tumorgenesis [5].With respect to the role in cancer, studies indicate that lncRNAs can modulate mRNA translation and decay in a base-pairing-dependent manner[6-8] or compete with a miRNA- or protein- mediated mRNA decoy[9]. Besides, it has was newly found that lncRNAs are involved in trafficking the cytoplasmic protein from cytosol to nucleus for transcriptional activation[10]. There are plentiful lncRNAs have been universally investigated and acknowledged as a biomarker of prognosis of cancer, including lncRNA CCAT1, HULC, MALAT1 and PVT1[11-14].Therefore, lncRNAs are regarded as promising markers for cancer diagnosis, prognosis and therapeutics[15].

The IncRNA Cancer Susceptibility Candidate 2(CASC2), which is located at chromosome 10q26, was firstly identified in endometrial cancer and its 
mRNA is downregulated in tumor tissues[16]. Abnormal CASC2 expression has been reported in diverse human cancers and play a role in regulating the progression of various cancers, including bladder cancer[17], renal cell carcinoma[18], esophageal carcinoma[19] and etc. Besides, plentiful studies have demonstrated that CASC2 aberrant expression is associated with prognosis of human tumors [20-30].However, a large proportion of individual studies are so confined in discrete and inconsonant outcome as well as small sample size. To our knowledge, no comprehensive meta-analysis has been conducted to discuss the relationship between lncRNA CASC2 and the correlative clinical outcomes of various carcinomas. In this, we conducted a quantitative meta-analysis to formulate the prognostic value of lncRNA CASC2 expression in cancer patients by systematically summarizing the qualified articles.

\section{Materials and Methods}

\section{Search strategy and literature selection}

Comprehensive literature search was performed in PubMed, Google Scholar, Web of Science, Cochrane Library, CNKI (China National Knowledge Infrastructure), PMC and Wanfang databases, and the cut-off date was defined as March 9, 2018. The searched terms in variably combinations were listed as follows: ("long noncoding RNA-" OR "lnc RNA-" OR "CASC2") and ("cancer" OR "carcinoma" OR "tumor" OR "neoplasm") and ("prognosis" OR "prognostic" OR "survival" OR "metastasis"). In addition, we manually reviewed the reference lists and contacted authors for further information when necessary.

\section{Inclusion and exclusion criteria}

The inclusion criteria were as follows: 1) Studies explored the clinical roles of CASC2 in different cancers; 2) research on the association of CASC2 expression level with prognosis or clinicopathological features; 3) patients were divided into two groups: high and low expression levels of CASC2.

Exclusion criteria for the articles included: 1) Studies without sufficient or available data; 2) Studies without dichotomous lncRNA CASC2 expression; 3) Duplicated studies; 4) Case reports, basic research, animal experiments, expert opinions, letters, reviews and conference abstracts.

\section{Data extraction and quality assessment}

Two reviewers independently extracted the data and information from the included studies. The following information were extracted from each eligible study: 1) first authors, year of publication, country, sample size, cancer type, follow-up time; 2) criteria for defining high and low expression level of CASC2, method for detecting CASC2 expression; 3) $\mathrm{HR}$ and its corresponding 95\% CI of lncRNA CACS2 for overall survival, the clinicopathological parameters including TNM stage, lymph node metastasis, tumor size and tumor differentiation. If studies only provided Kaplan-Meier curves, the survival rates were extracted from the graphical survival plots and the calculated $\mathrm{HR}$ and $95 \% \mathrm{CI}$ was reckoned following the published methods[31]. Multivariate analysis was preferred to extract because of higher precision when univariate and multivariate analysis were provided in the meantime. The quality of included studies was assessed according to Reporting Recommendations for Tumor Marker Prognostic Studies (REMARK) guideline (Supplementary Figure 1), the study scores ranged from 55\% to $70 \%$, and studies with more than $60 \%$ scores could be regarded as high quality. The detailed assessment was show in Supplementary Table 1.

\section{Statistical analysis}

1) The statistical analysis was performed with Stata SE12.0 (Stata Corporation) and RevMan 5.3 software. 2) HRs and 95\% CIs were used to assess the association of CASC2 expression with patients' prognosis. Odds Ratios (ORs) were performed to estimate the association of CASC2 expression with TNM stage, lymph node metastasis, tumor differentiation and tumor. If $\mathrm{HR}>1$, it represents that patients with high CASC2 expression have a poor prognosis. 3) Heterogeneity was quantified with Chi square-based $Q$ test and $I^{2}$ statistics. The effects model was adopted based on the heterogeneity, a fixed effects model was applied in if there was no significant heterogeneity, otherwise the random effects model was chosen. 4) The potential publication bias was estimated by the funnel plot and Begg's bias test $(P$ value $<0.05$ was considered statistically significant)[32]. In addition, the sensitivity analysis was also selected to assess the stability of the results.

\section{Results}

\section{Eligible literatures}

A total of 290 articles identified with the search strategy and were retrieved from several different databases. After ruling out the 178 duplicate publications, 112 papers were further carefully screened of titles, abstracts and full-texts, finally 11 articles were selected in the current meta-analysis due to meeting the inclusion criteria in methods. The selection process with more particulars was presented by a flow diagram in Figure 1. 


\section{Characteristics of included studies}

The main characteristics of 11 eligible articles were summarized in Table 1 . The studies were published between 2016 and 2018 and with a maximum size of 97 and a minimum size of 47 . The whole participants were classified into high and low CASC2 group in the light of qRT-PCR measurement results. All of studies come from China and there were 9 kinds of carcinomas in the midst of them, including non-small cell lung cancer, gastric cancer, lung adenocarcinoma, osteosarcoma, glioma, colorectal cancer, pituitary adenoma, hepatocellular cancer and thyroid cancer. It is worth noting that the median and mean values were applied as the cut-off value in articles.

\section{The association between CASC2 expression with prognosis of human cancers.}

Eight studies including 647 patients reported the $\mathrm{HR}$ and corresponding $95 \% \mathrm{CI}$ of OS. As indicated in Figure 2, enforced CASC2 expression was significantly correlated with better OS in various carcinomas (HR=0.43, 95\% CI: 0.33-0.55, $P=0.000$ ). There was no evidence of statistically heterogeneity between studies $\left(\mathrm{I}^{2}=0.0 \%\right)$. To maximize the clinical relevance, subgroups were analyzed in a fixed effects model by cancer types, sample sizes, study quality and follow-up months (Figure 3). For studies assessing OS in different types of cancer, the results indicated that promoted CASC2 could estimate preferable outcome in nervous system (HR:0.42, 95\% CI: $0.29-0.62, P=0.000)$, respiratory system (HR:0.39, 95\% CI: 0.21-0.73, $P=0.003$ ), digestive system (HR:0.51, 95\% CI: $0.31-0.86, \quad P=0.011)$, or other system malignancies (HR:0.35, 95\% CI: 0.17-0.72, $P=0.004$ ) (Figure 3A). In addition, there was a significant association between increased CASC2 expression and better OS in studies both with high quality (HR:0.38, 95\% CI: 0.26-0.55, $P=0.000$ ) and low quality (HR:0.47, 95\% CI: 0.34-0.66, $P=0.000$ ) (Figure 3B). Subsequently, we discovered that neither follow up time nor sample size altered the predictive value of CASC2 on the OS for all included cancers. (Figure 3C and 3D). No significant heterogeneity was found across studies within the subgroups.

\section{Correlation between CASC2 expression and clinical characteristics in patients with cancer}

We collected 11 researches consisting of 765 tumor samples to do a further meta-analysis for the association between CASC2 expression level and clinicopathological characteristics. As shown in Figure 4, the increased CASC2 expression was significantly associated with early TNM stage $(\mathrm{OR}=$ $0.30,95 \% \mathrm{CI}=0.21-0.43, P<0.001$, Figure $4 \mathrm{~A})$, smaller tumor size $(\mathrm{OR}=0.28,95 \% \mathrm{CI}=0.12-0.66, P=0.004$, Figure 4C), and better tumor differentiation (OR = $0.42,95 \% \mathrm{CI}=0.27-0.66, P=0.0002$, Figure $4 \mathrm{D}$ ). However, there was no significant association was found with lymph node metastasis (OR $=0.76,95 \% \mathrm{CI}$ $=0.48-1.20, P=0.24$, Figure 4B).

\section{Publication bias}

The potential publication bias was evaluated by funnel plot and Begg's bias test. The funnel plot was presented in Figure 5 and the shape of it was almost symmetrical. Moreover, the $P$ value of the Begg's test was 0.175 for OS of enrolled studies, suggesting a low risk of publication bias in meta-analysis.

\section{Sensitivity analysis}

To test the stability of meta-analysis of CASC2 and OS, we performed a sensitivity analysis and the result indicated that the results were stable because the pooled HR was not significantly impacted by the removing each single study (Figure 6).

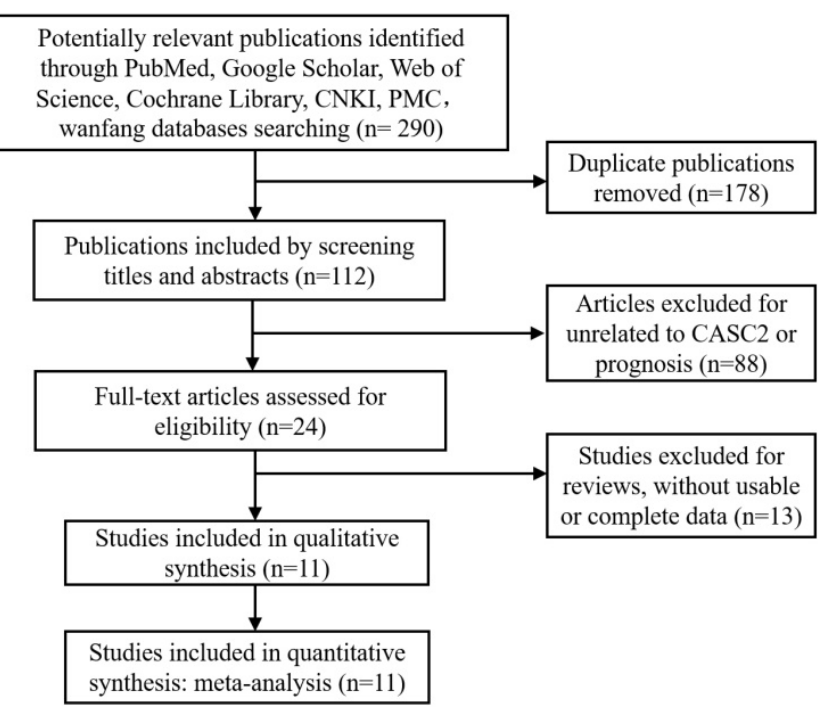

Figure 1. The flow diagram of study search and selection for meta-analysis.

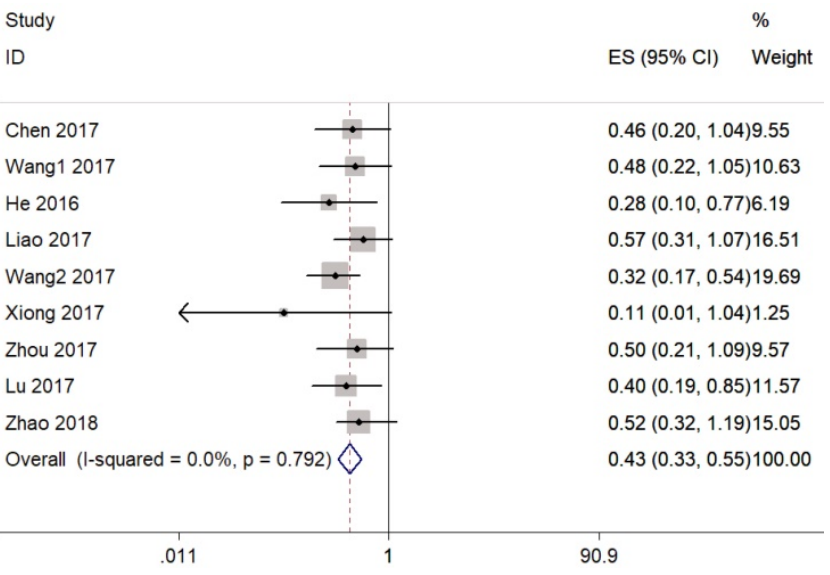

Figure 2. Forest plot for the association between CASC2 expression with overall survival (OS). 


\section{A}

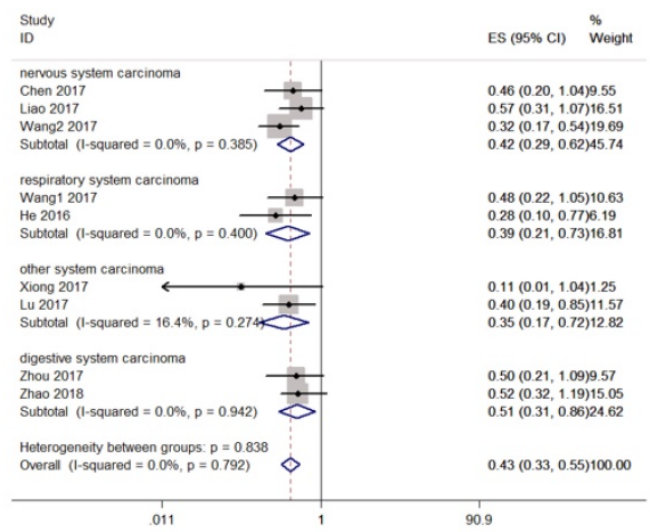

B
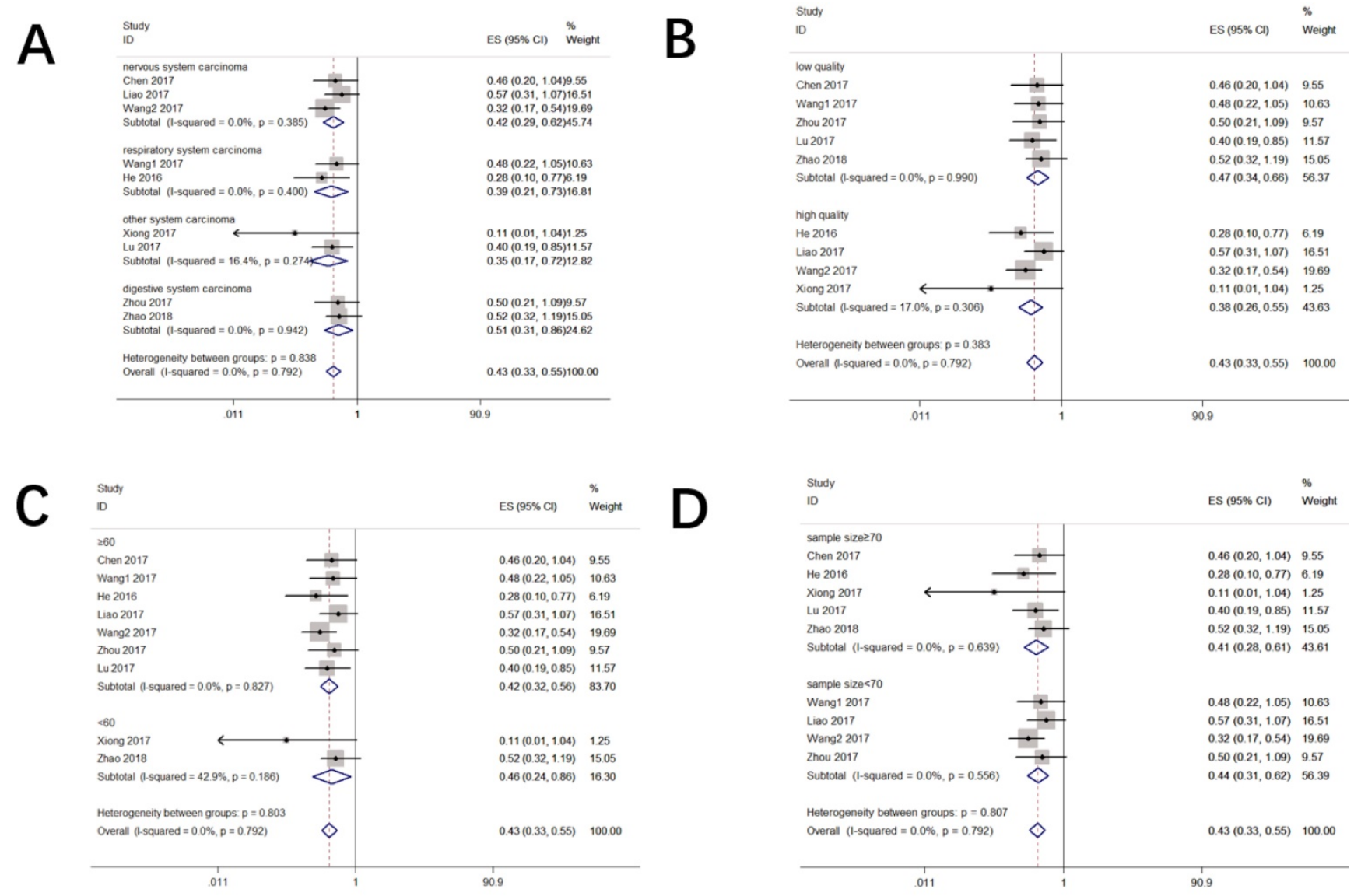

Figure 3. Stratified analyses for the association between CASC2 expression with overall survival (OS). (A) Subgroup analysis of HRs of OS by cancer type. (B) Subgroup analysis of HRs of OS by study quality. (C) Subgroup analysis of HRs of OS by follow up months. (D) Subgroup analysis of HRs of OS by sample size.

Table 1: Summary of included eligible studies for meta-analysis in the present study

\begin{tabular}{|c|c|c|c|c|c|c|c|c|c|c|c|}
\hline Author & Year & Region & Tumor type & Clinical stage & $\begin{array}{l}\text { Sample } \\
\text { size }\end{array}$ & CASC2 assay & $\begin{array}{l}\text { Cut-off } \\
\text { value }\end{array}$ & $\begin{array}{l}\text { Outcome } \\
\text { measure }\end{array}$ & $\begin{array}{l}\text { Hazard } \\
\text { ratios }\end{array}$ & $\begin{array}{l}\text { Follow-up } \\
\text { months }\end{array}$ & $\begin{array}{l}\text { Quality (estimate on } \\
\text { the REMARK } \\
\text { guideline }\end{array}$ \\
\hline Chen et al. [30] & 2017 & China & PA & I/II, III/IV & 72 & qRT-PCR & median & OS & KM & 60 & 55 \\
\hline Wang1 et al. [24] & 2017 & China & LA & I/II, III/IV & 63 & qRT-PCR & Median & OS & KM & 60 & 55 \\
\hline Gan et al. [29] & 2017 & China & $\mathrm{HCC}$ & I/II, III/IV & 50 & qRT-PCR & NA & NA & NA & NA & 55 \\
\hline He et al. [28] & 2016 & China & NSCLC & I/II, III/IV & 76 & qRT-PCR & mean & Os & $\mathrm{HR} / \mathrm{KM}$ & 60 & 65 \\
\hline Huang et al. [27] & 2016 & China & $\mathrm{CC}$ & I/II, III/IV & 68 & qRT-PCR & mean & NA & NA & NA & 60 \\
\hline Liao et al. [26] & 2017 & China & glioma & I/II, III/IV & 57 & qRT-PCR & median & OS & $\mathrm{HR} / \mathrm{KM}$ & 60 & 70 \\
\hline Wang2 et al. [23] & 2017 & China & glioma & I/II, III/IV & 47 & qRT-PCR & median & OS & $\mathrm{HR} / \mathrm{KM}$ & 60 & 70 \\
\hline Xiong et al. [22] & 2017 & China & $\mathrm{TC}$ & I/II, III/IV & 86 & qRT-PCR & mean & OS & $\mathrm{HR} / \mathrm{KM}$ & 32 & 70 \\
\hline Zhou et al. [21] & 2017 & China & GC & I/II, III/IV & 59 & qRT-PCR & mean & OS & $\mathrm{KM}$ & 70 & 60 \\
\hline Lu et al. [25] & 2017 & China & osteosarcoma & I/II, III/IV & 97 & qRT-PCR & median & OS & KM & 60 & 60 \\
\hline Zhao et al. [20] & 2018 & China & HCC & I/II, III/IV & 80 & qRT-PCR & NA & OS & KM & 50 & 55 \\
\hline
\end{tabular}

HR, hazard ratio; OS, overall survival; KM, Kaplan-Meier; qRT-PCR, quantitative real-time PCR; PA, pituitary adenoma; LA, lung adenocarcinoma; HCC, hepatocellular cancer; NSCLC, non-small lung cell cancer; CC, colorectal cancer; TC, thyroid cancer; GC, gastric cancer

\section{Discussion}

The growing evidence has demonstrated a myriad of functions for lncRNAs in tremendous cellular processes. Many lncRNAs have been shown to interfere with transcriptional machinery or be involved in maintaining the structure of nuclear speckles[33-35]. Furthermore, some lncRNAs act as regulators of post-transcriptionally splicing, protein stability, mRNA decay, or as molecular decoys for microRNAs[36, 37]. Owing to the specific expression pattern of lncRNA in the occurrence and development of carcinomas, lncRNAs can be regarded as a promising biomarker to monitor and diagnosis tumor, and they can be collected from body fluids and tissues readily[38]. 


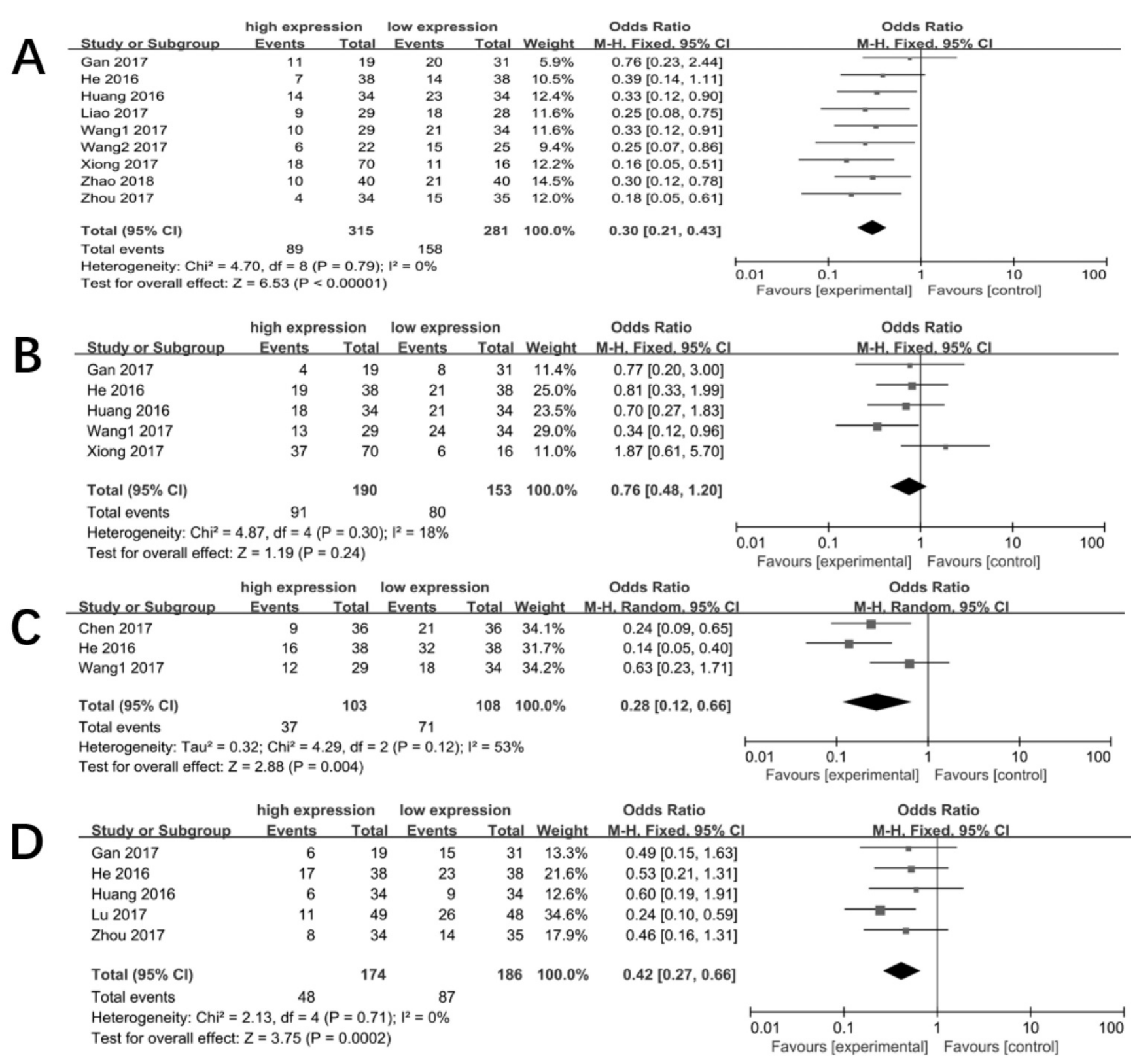

Figure 4. Forest plot for the association between CASC2 expression with TNM stage(A), lymph node metastasis(B), tumor size(C), tumor differentiation(D).

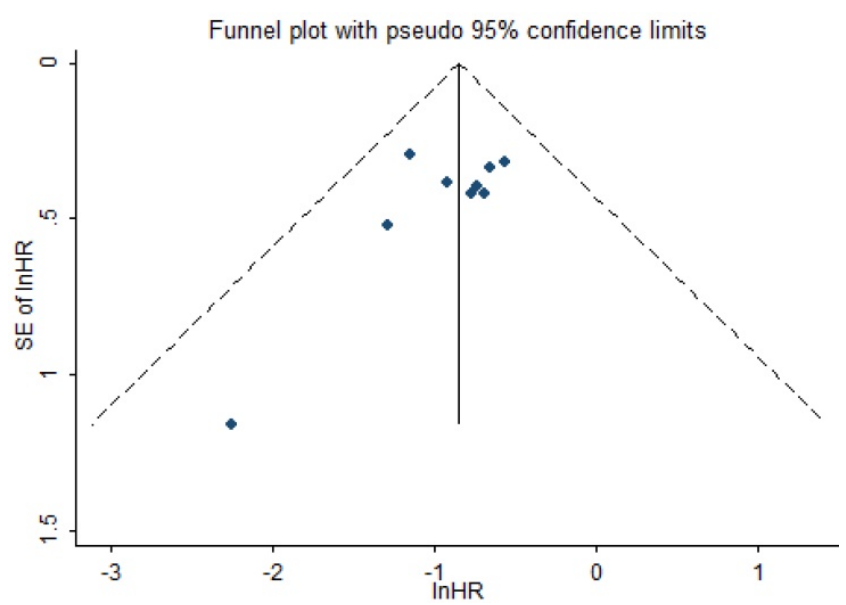

Figure 5. Funnel plot of the publication bias for OS.

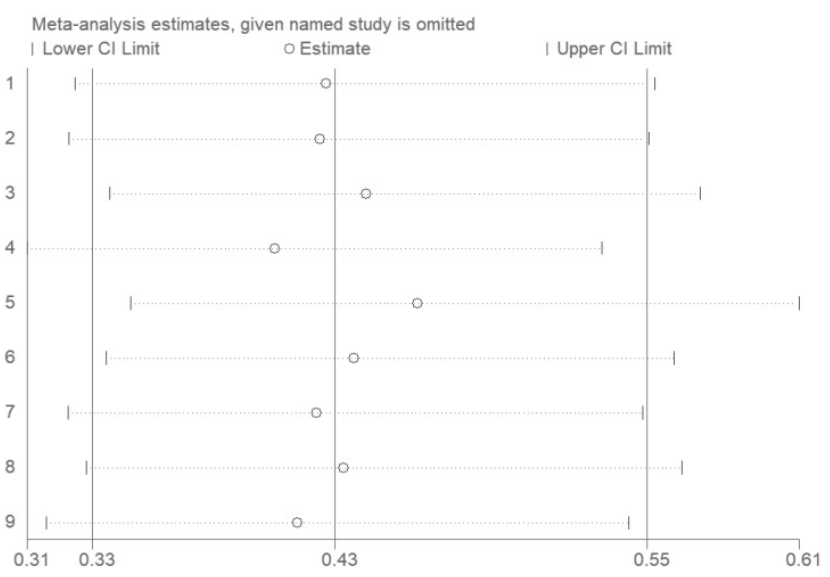

Figure 6. Sensitivity of CASC2 expression for OS 
Among the rising cancer-associated lncRNAs, CASC2 has been reported as a tumor suppressor and play crucial regulatory roles in in various carcinomas. For instance, lncRNA CASC2 inhibits cell proliferation and migration in renal cell carcinoma[18], hepatocellular cancer[29] and breast cancer[39]. In addition, it acts as a competing endogenous RNA(ceRNA) of miR-18a-5p in esophageal cancer and modulate the expression of PTEN[19]. In prostate cancer, CASC2 competes with SPRY2 for miR-183 binding and inhibit downstream extracellular regulated protein kinases (ERK) signaling[40]. Besides, CASC2 can promote PLXNC1 expression by sponging miR-181a, thereby inhibiting the proliferation and invasion of melanoma cells[41]. In TRAIL resistance hepatocellular carcinoma, CASC2 also acts as a "sponge" of miR-24 and miR-221, which contributes to improving the regent resistance[42]. As for the prognostic role, mounting studies elucidate the decreased expression of CASC2 in tumor tissues compares with normal tissues and high expression patients are prone to have a longer OS. In summary, the exist evidence implies the prognosis role of CASC2 in various carcinomas.

Up to now, the association between CASC2 and related clinic features is not clear even some is opposite, and the quality of present studies is not good enough. For instance, some studies lack multivariate analysis of clinic pathologic factors for overall survival[21, 25, 27], besides, some studies only provided a Kaplan-Meier curve, but didn't have a specific value of HR[20, 21].In addition, the sample of some study is too small[23].Thus, we generalized and analyzed the clinicopathologic significance and prognostic value of CASC2 in cancer patients. The present comprehensive meta-analysis pooled 11 independent studies with 765 cancer patients and the results indicated that enforced CASC2 pression was a protective factor for OS in cancer patients $(\mathrm{HR}=0.43$, $95 \%$ CI: $0.33-0.55, P=0.000$ ). In the subgroup analysis based on the system of cancer, it is demonstrated that CASC2 is a reliable prognostic biomarker for nervous system cancers, respiratory system cancers, digestive system cancers or other kinds of cancers. Likewise, the association between CASC2 and OS was significant in studies with high quality (HR:0.38, 95\% CI: 0.26-0.55, $\mathrm{P}=0.000$ ) or with low quality (HR:0.47, 95\% CI: $0.34-0.66, \mathrm{P}=0.000)$. In addition, the predictive value of CASC2 remained stable when we analyzed it separately in different sample size or follow up time. Collective studies also showed the correlation between CASC2 and clinicopathological parameters. Here, results showed that patients with relative high CASC2 expression had a lower risk of advanced TNM stage, larger tumor size and poor differentiation.
However, looking at results for lymph node, analysis failed to demonstrated a correlation with CASC2 expression, and it remain to be verified when pooled in more studies.

Nonetheless, the results of this meta-analysis should be treated with caution due to some unavoidable limitations. First, the number of included studies was small and cancer patients were all Chinese, which may lead to geographical bias. Second, some of HRs were calculated by survival curves so the value was not accurate. In addition, some studies didn't provide the cut off definition and the reported ones were not consistent. Finally, despite there was no obvious publication bias revealed, it is inevitable that works with non-significant results would have little chance to be published.

In conclusion, despite some limitations mentioned existed, the meta-analysis preliminarily offered conclusion that promoted CASC2 may be considered as a protective prognostic factor in human cancers. In the future, more well designed, large scale studies are required to support and strengthen the results of the present analysis.

\section{Supplementary Material}

Supplementary figures and tables.

http://www.jcancer.org/v09p3824s1.pdf

\section{Abbreviations}

LncRNA, long non-coding RNA; ncRNA, non-coding RNA; OS, overall survival; HR, hazard ratio; OR, odds ratios; $\mathrm{CI}$, confidence interval; $\mathrm{HCC}$, hepatocellular carcinoma; GC, gastric cancer; PA, pituitary adenoma; LA, lung adenocarcinoma; NSCLC, non-small cell lung cancer; CC, colorectal cancer; TC, thyroid cancer; REMARK, reporting recommendations for tumor marker prognostic studies.

\section{Acknowledgments}

This study was supported by the National Natural Science Foundation of China (Grant No. 81330002). The author would thank Dr. Yang Song in Pfizer medical for helpful suggestions.

\section{Author Contributions}

XY, MXL, YTZ conceived and designed the study. XY and WHS searched databases and extracted the data. JW, QQZ and QTW analyzed and checked the results of the data. FWL and LMC made some tables and figures. $X Y$ wrote the manuscript. All authors reviewed the final manuscript. 


\section{Competing Interests}

The authors have declared that no competing interest exists.

\section{References}

1. Siegel RL, Miller KD, Jemal A. Cancer statistics, 2018. CA: a cancer journal for clinicians. 2018; 68: 7-30.

2. Bray F, Jemal A, Grey N, Ferlay J, Forman D. Global cancer transitions according to the Human Development Index (2008-2030): a population-based study. The Lancet Oncology. 2012; 13: 790-801.

3. Borrebaeck CA. Precision diagnostics: moving towards protein biomarker signatures of clinical utility in cancer. Nature reviews Cancer. 2017; 17: 199-204

4. Esteller M. Non-coding RNAs in human disease. Nature reviews Genetics. 2011; 12: 861-74.

5. Isin M, Dalay N. LncRNAs and neoplasia. Clinica chimica acta; international journal of clinical chemistry. 2015; 444: 280-8

6. Kim YK, Furic L, Parisien M, Major F, DesGroseillers L, Maquat LE. Staufen1 regulates diverse classes of mammalian transcripts. The EMBO journal. 2007; 26: $2670-81$

7. Gong C, Maquat LE. IncRNAs transactivate STAU1-mediated mRNA decay by duplexing with 3' UTRs via Alu elements. Nature. 2011; 470: 284-8.

8. Beltran M, Puig I, Pena C, Garcia JM, Alvarez AB, Pena R, et al. A natural antisense transcript regulates Zeb2/Sip1 gene expression during Snail1-induced epithelial-mesenchymal transition. Genes \& development. 2008; 22: 756-69.

9. Cesana M, Cacchiarelli D, Legnini I, Santini T, Sthandier O, Chinappi M, et al. A long noncoding RNA controls muscle differentiation by functioning as a competing endogenous RNA. Cell. 2011; 147: 358-69.

10. Liu B, Sun L, Liu Q, Gong C, Yao Y, Lv X, et al. A cytoplasmic NF-kappaB interacting long noncoding RNA blocks IkappaB phosphorylation and suppresses breast cancer metastasis. Cancer cell. 2015; 27: 370-81.

11. Liu F, Dong Q, Huang J. Overexpression of LncRNA PVT1 Predicts Advanced Clinicopathological Features and Serves as an Unfavorable Risk Factor for Survival of Patients with Gastrointestinal Cancers. Cellular physiology and biochemistry : international journal of experimental cellular physiology, biochemistry, and pharmacology. 2017; 43: 1077-89.

12. Guo X, Hua Y. CCAT1: an oncogenic long noncoding RNA in human cancers. Journal of cancer research and clinical oncology. 2017; 143: 555-62.

13. Chen $X$, Lun L, Hou $H$, Tian $R$, Zhang $H$, Zhang $Y$. The Value of lncRNA HULC as a Prognostic Factor for Survival of Cancer Outcome: A Meta-Analysis. Cellular physiology and biochemistry : international journal of experimental cellular physiology, biochemistry, and pharmacology. 2017; 41: 1424-34.

14. Li Z, Li J, Tang N. Long noncoding RNA Malat1 is a potent autophagy inducer protecting brain microvascular endothelial cells against oxygen-glucose deprivation/reoxygenation-induced injury by sponging miR-26b and upregulating ULK2 expression. Neuroscience. 2017; 354: 1-10.

15. Tsai MC, Spitale RC, Chang HY. Long intergenic noncoding RNAs: new links in cancer progression. Cancer research. 2011; 71: 3-7.

16. Baldinu P, Cossu A, Manca A, Satta MP, Sini MC, Rozzo C, et al. Identification of a novel candidate gene, CASC2, in a region of common allelic loss at chromosome 10q26 in human endometrial cancer. Human mutation. 2004; 23 : 318-26.

17. Pei Z, Du X, Song Y, Fan L, Li F, Gao Y, et al. Down-regulation of lncRNA CASC2 promotes cell proliferation and metastasis of bladder cancer by activation of the Wnt/beta-catenin signaling pathway. Oncotarget. 2017; 8: 18145-53.

18. Cao Y, Xu R, Xu X, Zhou Y, Cui L, He X. Downregulation of IncRNA CASC2 by microRNA-21 increases the proliferation and migration of renal cell carcinoma cells. Molecular medicine reports. 2016; 14: 1019-25.

19. Zhang W, He W, Gao J, Wang Y, Zang W, Dong Z, et al. The long noncoding RNA CASC2 inhibits tumorigenesis through modulating the expression of PTEN by targeting miR-18a-5p in esophageal carcinoma. Experimental cell research. 2017; 361: 30-8.

20. Zhao L, Zhang Y. Long noncoding RNA CASC2 regulates hepatocellular carcinoma cell oncogenesis through miR-362-5p/Nf-kappaB axis. Journal of cellular physiology. 2018

21. Zhou J, Huang H, Tong S, Huo R. Overexpression of long non-coding RNA cancer susceptibility 2 inhibits cell invasion and angiogenesis in gastric cancer. Molecular medicine reports. 2017; 16: 5235-40.

22. Xiong $\mathrm{X}, \mathrm{Zhu} \mathrm{H}$, Chen $\mathrm{X}$. Low expression of long noncoding RNA CASC2 indicates a poor prognosis and promotes tumorigenesis in thyroid carcinoma. Biomedicine \& pharmacotherapy = Biomedecine \& pharmacotherapie. 2017; 93: 391-7.

23. Wang R, Li Y, Zhu G, Tian B, Zeng W, Yang Y, et al. Long noncoding RNA CASC2 predicts the prognosis of glioma patients and functions as a suppressor for gliomas by suppressing Wnt/beta-catenin signaling pathway. Neuropsychiatric disease and treatment. 2017; 13: 1805-13.

24. Wang D, Gao ZM, Han LG, Xu F, Liu K, Shen Y. Long noncoding RNA CASC2 inhibits metastasis and epithelial to mesenchymal transition of lung adenocarcinoma via suppressing SOX4. European review for medical and pharmacological sciences. 2017; 21: 4584-90.

25. Lu L, Dai Z, Luo Q, Lv G. The long noncoding RNA cancer susceptibility candidate 2 inhibits tumor progression in osteosarcoma. Molecular medicine reports. 2018; 17: 1947-53

26. Liao $Y$, Shen L, Zhao H, Liu Q, Fu J, Guo Y, et al. LncRNA CASC2 Interacts With miR-181a to Modulate Glioma Growth and Resistance to TMZ Through PTEN Pathway. Journal of cellular biochemistry. 2017; 118: 1889-99.

27. Huang G, Wu X, Li S, Xu X, Zhu H, Chen X. The long noncoding RNA CASC2 functions as a competing endogenous RNA by sponging miR-18a in colorectal cancer. Scientific reports. 2016; 6: 26524

28. He X, Liu Z, Su J, Yang J, Yin D, Han L, et al. Low expression of long noncoding RNA CASC2 indicates a poor prognosis and regulates cell proliferation in non-small cell lung cancer. Tumour biology : the journal of the International Society for Oncodevelopmental Biology and Medicine. 2016; 37: 9503-10

29. Gan Y, Han N, He X, Yu J, Zhang M, Zhou Y, et al. Long non-coding RNA CASC2 regulates cell biological behaviour through the MAPK signalling pathway in hepatocellular carcinoma. Tumour biology : the journal of the International Society for Oncodevelopmental Biology and Medicine. 2017; 39: 1010428317706229

30. <ijcem0055736.pdf>

31. Tierney JF, Stewart LA, Ghersi D, Burdett S, Sydes MR. Practical methods for incorporating summary time-to-event data into meta-analysis. Trials. 2007; 8:

32. Egger M, Davey Smith G, Schneider M, Minder C. Bias in meta-analysis detected by a simple, graphical test. BMJ (Clinical research ed). 1997; 315: 629-34.

33. Prasanth KV, Prasanth SG, Xuan Z, Hearn S, Freier SM, Bennett CF, et al. Regulating gene expression through RNA nuclear retention. Cell. 2005; 123: 249-63.

34. Clemson CM, Hutchinson JN, Sara SA, Ensminger AW, Fox AH, Chess A, et al. An architectural role for a nuclear noncoding RNA: NEAT1 RNA is essential for the structure of paraspeckles. Molecular cell. 2009; 33: 717-26.

35. Sunwoo H, Dinger ME, Wilusz JE, Amaral PP, Mattick JS, Spector DL. MEN epsilon/beta nuclear-retained non-coding RNAs are up-regulated upon muscle differentiation and are essential components of paraspeckles. Genome research. 2009; 19: 347-59.

36. Yoon JH, Abdelmohsen K, Gorospe M. Posttranscriptional gene regulation by long noncoding RNA. Journal of molecular biology. 2013; 425: 3723-30.

37. Quinn JJ, Chang HY. Unique features of long non-coding RNA biogenesis and function. Nature reviews Genetics. 2016; 17: 47-62.

38. Qi P, Du X. The long non-coding RNAs, a new cancer diagnostic and therapeutic gold mine. Modern pathology : an official journal of the United States and Canadian Academy of Pathology, Inc. 2013; 26: 155-65.

39. Zhang Y, Zhu M, Sun Y, Li W, Wang Y, Yu W. Up-regulation of IncRNA CASC2 suppresses cell proliferation and metastasis of breast cancer via inactivating of the TGF-ss signaling pathway. Oncology research. 2018.

40. Gao W, Lin S, Cheng C, Zhu A, Hu Y, Shi Z, et al. Long non-coding RNA CASC2 regulates Sprouty2 via functioning as a competing endogenous RNA for miR-183 to modulate the sensitivity of prostate cancer cells to docetaxel. Archives of biochemistry and biophysics. 2018

41. Wang Z, Wang X, Zhou H, Dan X, Jiang L, Wu Y. Long non-coding RNA CASC2 inhibits tumorigenesis via the miR-181a/PLXNC1 axis in melanoma. Acta biochimica et biophysica Sinica. 2018.

42. Jin X, Cai L, Wang C, Deng X, Yi S, Lei Z, et al. CASC2/miR-24/miR-221 modulates the TRAIL resistance of hepatocellular carcinoma cell through caspase-8/caspase-3. Cell death \& disease. 2018; 9: 318 